OPEN ACCESS

Edited by:

Jia-Bao Liu,

Anhui Jianzhu University, China

Reviewed by:

Anouar Ben Mabrouk,

University of Kairouan, Tunisia

Song Zheng,

Zhejiang University of Finance and

Economics, China

Xiaoyang Liu,

Jiangsu Normal University, China

*Correspondence:

Yongqing Wu

yqwuyywu@163.com

Specialty section:

This article was submitted to Mathematical and Statistical Physics,

a section of the journal

Frontiers in Physics

Received: 11 June 2020 Accepted: 08 September 2020

Published: 16 October 2020

Citation:

Zhang $L$ and Wu Y (2020)

Synchronizability of Multilayer Networks With K-nearest-neighbor Topologies. Front. Phys. 8:571507. doi: 10.3389/fphy.2020.571507

\section{Synchronizability of Multilayer Networks With K-nearest-neighbor Topologies}

\author{
Li Zhang ${ }^{1}$ and Yongqing $W u^{2 *}$ \\ ${ }^{1}$ Department of Mathematics, College of Science, Liaoning Technical University, Fuxin, China, ${ }^{2}$ Basic Teaching Department, \\ Liaoning Technical University, Huludao, China
}

In this paper, the synchronizability of multilayer K-nearest-neighbor networks is studied by using the master stability function method. The analytical expressions for the eigenvalues of the supra-Laplacian matrix are given for two-layer and multilayer K-nearest-neighbor networks. In addition, the impacts of various topological parameters (such as the network size, the node degree, the number of layers, the intra-layer and the inter-layer coupling strengths) on the network synchronizability are discussed. Finally, the theoretical results are verified through numerical simulation.

Keywords: multilayer network, K-nearest-neighbor topology, synchronizability, master stability function method, supra-Laplacian matrix

\section{INTRODUCTION}

Since the appearance of small-world networks and scale-free networks [1, 2], complex networks have attracted much attention due to their pervading through various scientific fields. Till now, complex networks have been applied in nature and society, such as scientific cooperation networks, information networks, biological networks, power grids, social networks, and so on [3, 4]. Recently, a new description of the complex network called the multilayer network, where nodes interact with more than one type of links, was put forward and gradually became an important branch of complex networks [5-8].

Synchronization, as a significative collective behavior on complex networks, has been widely and extensively discussed during the past two decades [9-14]. Further, there has been an increasing interest focusing on the finite-time synchronization, especially when the synchronization is required to be realized in finite time because of practical need $[15,16]$. On the other hand, scholars have done a lot of work to analyze the synchronization of multilayer networks. A general framework for studying the diffusion processes on multiplex networks was proposed in [17, 18]. In 2014, Aguirre et al. revealed that the connector nodes between layers play an important role in the synchronizability of interconnected networks [19]. Further, intra-layer synchronization, inter-layer synchronization, counterpart synchronization, and generalized synchronization in multiplex networks have been investigated [20-24]. Tang et al. proposed three necessary regions to describe the different types of coherent behaviors (such as complete synchronization, intra-layer synchronization, and inter-layer synchronization) in multiplex networks based on the master stability function method [25].

However, most of the existing works focused on the effects of network structures on the synchronizability of multilayer networks through numerical simulation. To better understand the relationships between topological parameters and synchronizability, it is necessary to give a more rigorous theoretical analysis. Recently, the analytical expressions for the eigenvalues of multilayer 
fully-connected networks, star networks, chain networks, and star-ring networks were derived to analyze the synchronizability [26-30]. To the best of our knowledge, very little work has been devoted to studying the synchronizability of multilayer K-nearest-neighbor networks. Due to the complexity of the multilayer networks, it is still a real challenge to derive the analytical expressions for the eigenvalue spectrum of the supraLaplacian matrix.

Motivated by the above discussion, we study the synchronizability of multilayer networks with K-nearestneighbor topologies. The present study uses the master stability function method to investigate the relationships between various topological parameters and network synchronizability. With this framework, we strictly derive the analytical expressions for the eigenvalues of two-layer and multilayer K-nearest-neighbor networks. Analytical and numerical results show that the network size, the node degree, the number of layers, the intra-layer and the inter-layer coupling strengths can have important effects on the synchronizability of multilayer K-nearest-neighbor networks.

The structure of this paper is organized as follows. The model of multilayer networks and some preliminaries are given in section 2. Section 3 studies the synchronizability of two-layer and multilayer K-nearest-neighbor networks. Numerical examples in section 4 illustrate the effectiveness of theoretical results. The conclusion is finally drawn in section 5 .

\section{PROBLEM FORMULATION AND PRELIMINARIES}

The dynamics of multilayer networks consisting of $M$ layers are described as follows [25]:

$$
\frac{d X_{i}^{\alpha}}{d t}=f\left(X_{i}^{\alpha}\right)-a \sum_{j=1}^{N} w_{i j}^{\alpha} H\left(X_{j}^{\alpha}\right)-d \sum_{\beta=1}^{M} d_{i}^{\alpha \beta} \Gamma\left(X_{i}^{\beta}\right)
$$

where $X_{i}^{\alpha} \in R^{n}$ is the state of the $i$-th node in the $\alpha$-th layer, $1 \leq i \leq N, 1 \leq \alpha \leq M \cdot f: R^{n} \rightarrow R^{n}$ is a smooth nonlinear vector function. The continuous function $H: R^{n} \rightarrow R^{n}$ and $a$ are the intra-layer coupling function and coupling strength, respectively. $\Gamma: R^{n} \rightarrow R^{n}$ and $d$ are the inter-layer coupling function and coupling strength, respectively. For simplicity, let $H\left(X_{j}^{\alpha}\right)=H X_{j}^{\alpha}, \Gamma\left(X_{i}^{\alpha}\right)=\Gamma X_{i}^{\alpha}$ and $H=\Gamma$. In the $\alpha$-th layer, if the $i$-th node is connected with the $j$-th $(j \neq i)$ node, $w_{i j}{ }^{\alpha}=-1$, otherwise, $w_{i j}{ }^{\alpha}=0$, and $w_{i i}{ }^{\alpha}=-\sum_{j=1, j \neq i}^{N} w_{i j}{ }^{\alpha}(i, j=1,2, \ldots, N$ and $\alpha=1,2, \ldots, M) . L^{(\alpha)}=\left(a w_{i j}{ }^{\alpha}\right) \in R^{N \times N}$ is the Laplacian matrix of the $\alpha$-th layer. Similarly, if the $i$-th node, in the $\alpha$-th layer, is connected with its replica in the $\beta$-th $(\alpha \neq \beta)$ layer, $d_{i}^{\alpha \beta}=-1$, otherwise $d_{i}^{\alpha \beta}=0$, and $d_{i}{ }^{\alpha \alpha}=-\sum_{k=1, k \neq \alpha}^{M} d_{i}{ }^{\alpha k}$ $(\alpha, \beta=1,2, \ldots, M(\alpha \neq \beta))$. It is obvious that $L^{I}=\left(d d_{i}^{\alpha \beta}\right) \in$ $R^{M \times M}$ is the interlayer Laplacian matrix.
Denote

$$
\begin{gathered}
X^{(\alpha)}=\left(\begin{array}{c}
X_{1}{ }^{\alpha} \\
X_{2}{ }^{\alpha} \\
\vdots \\
X_{N}{ }^{\alpha}
\end{array}\right), X=\left(\begin{array}{c}
X^{(1)} \\
X^{(2)} \\
\vdots \\
X^{(M)}
\end{array}\right), \\
\tilde{f}\left(X^{(\alpha)}\right)=\left(\begin{array}{c}
f\left(X_{1}^{\alpha}\right) \\
f\left(X_{2}{ }^{\alpha}\right) \\
\vdots \\
f\left(X_{N}{ }^{\alpha}\right)
\end{array}\right), F(X)=\left(\begin{array}{c}
\tilde{f}\left(X^{(1)}\right) \\
\tilde{f}\left(X^{(2)}\right) \\
\vdots \\
\tilde{f}\left(X^{(M)}\right)
\end{array}\right),
\end{gathered}
$$

then we can rewrite the evolution of the multilayer network (1) as following form:

$$
\frac{d X}{d t}=F(X)-\left(\left(\mathcal{L}^{L}+\mathcal{L}^{I}\right) \otimes \Gamma\right) X
$$

where

$$
\begin{gathered}
\mathcal{L}^{L}=\left(\begin{array}{cccc}
L^{(1)} & 0 & \cdots & 0 \\
0 & L^{(2)} & \cdots & 0 \\
\vdots & \vdots & \ddots & \vdots \\
0 & 0 & \cdots & L^{(M)}
\end{array}\right)=\underset{\alpha=1}{\oplus} L^{(\alpha)}, \\
\mathcal{L}^{I}=L^{I} \otimes I_{N} .
\end{gathered}
$$

Here, $\otimes$ is the Kronecker product, $\oplus$ is the direct sum operation, $I_{N}$ is the $N \times N$ identity matrix. Then we can get the supraLaplacian matrix of multilayer networks (1),

$$
\mathcal{L}=\mathcal{L}^{L}+\mathcal{L}^{I}
$$

The decomposition of the supra-Laplacian matrix given in Equation (5) is fundamental for the discovery of several spectral performances of the multilayer networks [17]. According to the master stability function (MSF) framework [31], the synchronized regions of dynamical systems can be classified into four classes: empty, bounded, unbounded and the union of some bounded and unbounded regions. Here, we only focus on the bounded and unbounded synchronized regions. If the synchronized region is unbounded, the network synchronizability is positively correlated to the non-zero minimum eigenvalue $\left(\lambda_{2}\right)$ of the supra-Laplacian matrix. On the other hand, if the synchronized region is bounded, the eigenratio of the maximum eigenvalue and the non-zero minimum eigenvalue $\left(r=\lambda_{\max } / \lambda_{2}\right)$ characterizes the network synchronizability. A smaller $r$ value means that there is a stronger synchronizability of the network.

Throughout the rest of this paper, a useful lemma is presented as follows.

Lemma 1. (see [28]) Let $A$ and $B$ are two square matrices with the same size, then

$$
\left|\begin{array}{cccc}
A & B & \cdots & B \\
B & A & \cdots & B \\
\vdots & \vdots & \ddots & \vdots \\
B & B & \cdots & A
\end{array}\right|_{M \times M}=|A-B|^{M-1} \cdot|A+(M-1) B|,
$$

where $M$ is a positive integer greater than 1 . 


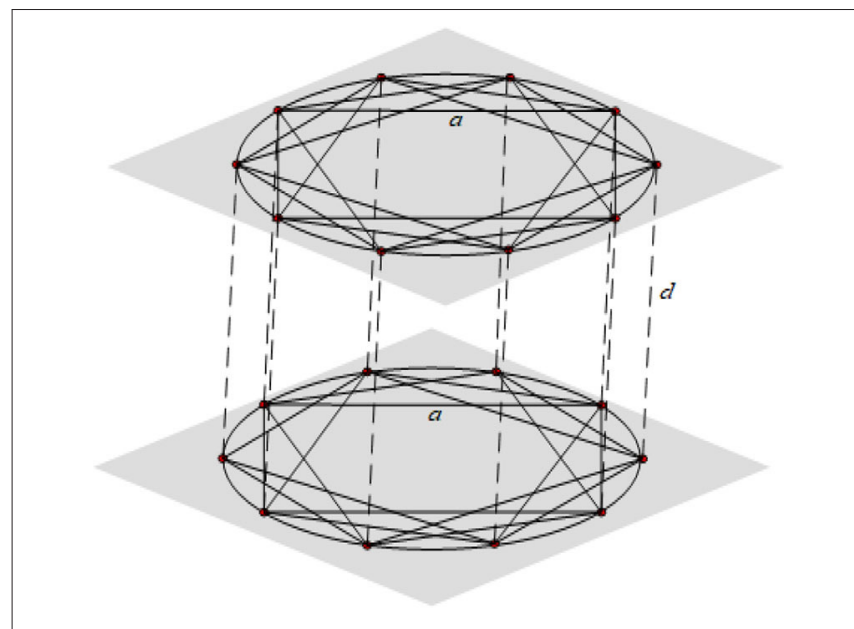

FIGURE 1 | Two-layer networks with K-nearest-neighbor topologies, where $d$ is the inter-layer coupling strength and $a$ is the intra-layer coupling strength.

\section{SYNCHRONIZABILITY OF MULTILAYER NETWORKS}

In this section, analytical results are presented for the synchronizability of multilayer networks with K-nearest-neighbor topologies.

\subsection{Two-Layer K-nearest-neighbor Networks}

We consider a two-layer model, each layer is a K-nearestneighbor network with $\mathrm{N}$ nodes ( $\mathrm{K}$ is an even number) and every node in one layer is connecting with its counterpart in the other layer. The corresponding structure can be shown in Figure 1.

So the supra-Laplacian matrix can be written as follows:

$$
\mathcal{L}=\left(\begin{array}{cc}
A+d I_{N} & -d I_{N} \\
-d I_{N} & A+d I_{N}
\end{array}\right)
$$

where

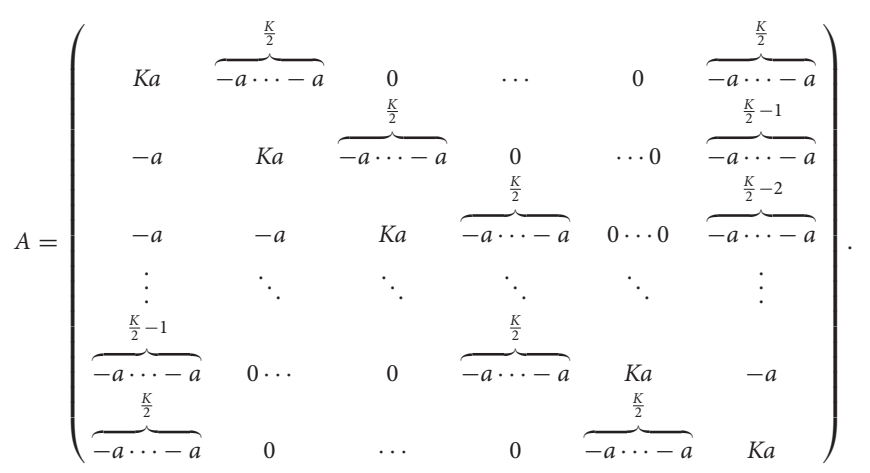

TABLE 1 | Change of $a, d, K, N$ for $\lambda_{2}, r=\lambda_{\max } / \lambda_{2}$ of two-layer

K-nearest-neighbor networks.

\begin{tabular}{|c|c|c|c|c|c|c|}
\hline & & Increase of & $a$ & $d$ & $K$ & $N$ \\
\hline$\lambda_{2}$ & $\begin{array}{l}d<\frac{a \pi^{2} K(K+1)(K+2)}{12 N^{2}} \\
d>\frac{a \pi^{2} K(K+1)(K+2)}{12 N^{2}}\end{array}$ & $\begin{array}{c}\lambda_{2} \approx 2 d \\
\lambda_{2} \approx \frac{a \pi^{2} K(K+1)(K+2)}{6 N^{2}}\end{array}$ & $\begin{array}{l}- \\
\uparrow\end{array}$ & $\begin{array}{l}\uparrow \\
-\end{array}$ & $\begin{array}{l}- \\
\uparrow\end{array}$ & $\begin{array}{l}- \\
\downarrow\end{array}$ \\
\hline$r=\frac{\lambda_{\max }}{\lambda_{2}}$ & $\begin{array}{l}d<\frac{a \pi^{2} K(K+1)(K+2)}{12 N^{2}} \\
d>\frac{a \pi^{2} K(K+1)(K+2)}{12 N^{2}}\end{array}$ & $\begin{array}{c}r \approx \frac{a(K+1)(3 \pi+2)+6 \pi d}{6 \pi d} \\
r \approx \frac{2 a N^{2}(K+1)(3 \pi+2)+12 \pi d N^{2}}{a \pi^{3} K(K+1)(K+2)}\end{array}$ & $\begin{array}{l}\uparrow \\
\downarrow\end{array}$ & $\begin{array}{l}\downarrow \\
\uparrow\end{array}$ & $\begin{array}{l}\uparrow \\
\downarrow\end{array}$ & $\begin{array}{l}- \\
\uparrow\end{array}$ \\
\hline
\end{tabular}

$\uparrow$, increase; $\downarrow$, decrease; - , unchange.

According to Lemma 1, we can get the characteristic polynomial of $\mathcal{L}$ :

$$
\begin{aligned}
\left|\lambda I_{2 N}-\mathcal{L}\right| & =\left|\begin{array}{cc}
\lambda I_{N}-\left(A+d I_{N}\right) & d I_{N} \\
d I_{N} & \lambda I_{N}-\left(A+d I_{N}\right)
\end{array}\right| \\
& =\left|(\lambda-2 d) I_{N}-A\right| \cdot\left|\lambda I_{N}-A\right| .
\end{aligned}
$$

Let $\left|\lambda I_{N}-A\right|=0$, the eigenvalues of the K-nearest neighbor network can be written as [32]:

$$
\lambda_{l}=K a-2 a \sum_{j=1}^{K / 2} \cos \left(\frac{2 \pi(l-1) j}{N}\right), \quad l=1,2, \ldots, N .
$$

Let $\left|(\lambda-2 d) I_{N}-A\right|=0$, it follows that

$$
\lambda_{l}-2 d=K a-2 a \sum_{j=1}^{K / 2} \cos \left(\frac{2 \pi(l-1) j}{N}\right), \quad l=1,2, \ldots, N .
$$

Then, the eigenvalues of $\mathcal{L}$ are

$$
\begin{aligned}
0, K a & -2 a \sum_{j=1}^{K / 2} \cos \left(\frac{2 \pi(l-1) j}{N}\right), 2 d, K a \\
& +2 d-2 a \sum_{j=1}^{K / 2} \cos \left(\frac{2 \pi(l-1) j}{N}\right), \\
l & =2,3, \ldots, N .
\end{aligned}
$$

When $1 \ll K \ll N$, we can obtain the smallest non-zero eigenvalue $\lambda_{2}$ and the largest eigenvalue $\lambda_{\max }$ based on the series expansion,

$$
\begin{aligned}
\lambda_{2} & \approx \min \left\{\frac{a \pi^{2} K(K+1)(K+2)}{6 N^{2}}, 2 d\right\}, \\
\lambda_{\max } & \approx a(K+1)\left(1+\frac{2}{3 \pi}\right)+2 d .
\end{aligned}
$$

The relationships between $\lambda_{2}, r=\lambda_{\max } / \lambda_{2}$ and the network parameters are shown in Table 1.

Remark 1. When $K=2$, the eigenvalues of single networks are $\lambda_{l}=2 a-2 a \cos \left(\frac{2 \pi(l-1)}{N}\right)=4 a \sin ^{2}\left(\frac{(l-1) \pi}{N}\right), l=$ $1,2, \ldots, N$. Then, the eigenvalues of supra-Laplacian matrix $\mathcal{L}$ are $4 a \sin ^{2}\left(\frac{(l-1) \pi}{N}\right), 2 d+4 a \sin ^{2}\left(\frac{(l-1) \pi}{N}\right), l=1,2, \ldots, N$. In [26], synchronizability of duplex ring networks was investigated. Obviously, the network model of this paper is more general. 


\subsection{Multilayer K-nearest-neighbor Networks}

Similarly, we consider the multilayer network with M layers, the corresponding structure is shown in Supplementary Figure 1.

We obtain the supra-Laplacian matrix $\mathcal{L}$,

$$
\mathcal{L}=\left(\begin{array}{cccc}
A+(M-1) d I_{N} & -d I_{N} & \cdots & -d I_{N} \\
-d I_{N} & A+(M-1) d I_{N} & \cdots & -d I_{N} \\
\vdots & \vdots & \ddots & \vdots \\
-d I_{N} & -d I_{N} & \cdots & A+(M-1) d I_{N}
\end{array}\right)_{M \times M},
$$

where $A$ is given in section 3.1.

According to Lemma 1, the characteristic polynomial of $\mathcal{L}$ is:

$$
\begin{aligned}
& \left|\lambda I_{M N}-\mathcal{L}\right| \\
= & \left|\begin{array}{cccc}
(\lambda-(M-1) d) I_{N}-A & d I_{N} & \cdots & d I_{N} \\
d I_{N} & (\lambda-(M-1) d) I_{N}-A & \cdots & d I_{N} \\
\vdots & \vdots & \ddots & \vdots \\
d I_{N} & d I_{N} & \cdots & (\lambda-(M-1) d) I_{N}-A
\end{array}\right| \\
= & \left|(\lambda-M d) I_{N}-A\right|^{M-1} \cdot\left|\lambda I_{N}-A\right| .
\end{aligned}
$$

Then, the eigenvalues of $\mathcal{L}$ are

$$
\begin{aligned}
& 0, K a-2 a \sum_{j=1}^{K / 2} \cos \left(\frac{2 \pi(l-1) j}{N}\right), \underbrace{M d, \ldots, M d}_{M-1}, \\
& K a+M d-2 a \sum_{j=1}^{K / 2} \cos \left(\frac{2 \pi(l-1) j}{N}\right), \ldots, K a+M d \\
& -2 a \sum_{j=1}^{K / 2} \cos \left(\frac{2 \pi(l-1) j}{N}\right) \\
& M-1 \\
& l=2,3, \ldots, N \text {. }
\end{aligned}
$$

When $1 \ll K \ll N$, we can obtain the smallest non-zero eigenvalue $\lambda_{2}$ and the largest eigenvalue $\lambda_{\max }$ based on the series expansion,

$$
\begin{aligned}
\lambda_{2} & \approx \min \left\{\frac{a \pi^{2} K(K+1)(K+2)}{6 N^{2}}, M d\right\}, \\
\lambda_{\max } & \approx a(K+1)\left(1+\frac{2}{3 \pi}\right)+M d .
\end{aligned}
$$

The relationships between $\lambda_{2}, r=\lambda_{\max } / \lambda_{2}$ and the network parameters are shown in Supplementary Table 1.

Remark 2. When $M=2$, the smallest non-zero eigenvalue $\lambda_{2} \approx$ $\min \left\{\frac{a \pi^{2} K(K+1)(K+2)}{6 N^{2}}, 2 d\right\}$ and the largest eigenvalue $\lambda_{\max } \approx$ $a(K+1)\left(1+\frac{2}{3 \pi}\right)+2 d$, which are equal to the eigenvalues in Equations (10) and (11). Actually, the two-layer network is a special case of the multilayer network.

\section{NUMERICAL SIMULATIONS}

In this section, numerical examples are presented to study the synchronizability of the multilayer K-nearest-neighbor networks.

\subsection{The Synchronizability of Two-Layer Networks}

(1) Let $N=200, K=50, d=1$, the impact of the intra-layer coupling strength $a$ on network synchronizability is shown in Figures $\mathbf{2 A , B}$. When the synchronized region is unbounded, Figure $\mathbf{2 A}$ displays that $\lambda_{2}$ increases with small $a\left(a<a_{0}=\frac{12 N^{2} d}{\pi^{2} K(K+1)(K+2)} \approx 0.3668\right)$, and then reaches a certain value $\lambda_{2}=2 d=2$. This implies that the synchronizability of two-layer networks is first enhanced with small values of increasing $a$ and then held constant with ever-increasing $a$. When the synchronized region is bounded, it can be observed from Figure 2B that the eigenratio $r=\lambda_{\max } / \lambda_{2}$ first decreases with small $a\left(a<a_{0}\right)$ and then increases monotonically. It means that the synchronizability is enhanced firstly, then gets weakened after reaching the maximum. The synchronizability of two-layer networks is maximized at $a_{0}=\frac{12 N^{2} d}{\pi^{2} K(K+1)(K+2)}$.

(2) Let $N=200, K=50, a=0.5$, the impact of the inter-layer coupling strength $d$ on network synchronizability is shown in Figures 2C,D. When $0.5<d<d_{0}=$ $\frac{a \pi^{2} K(K+1)(K+2)}{12 N^{2}} \approx 1.3632$, Figure 2C depicts that $\lambda_{2}$ increases linearly with small $d$. When $d>d_{0}$, it reaches a certain value $\lambda_{2}=2 d_{0}$. This implies that, with an unbounded synchronized region, the synchronizability is first enhanced with small $d$ and then kept invariant. In Figure 2D, it can be observed that the eigenratio $r=$ $\lambda_{\max } / \lambda_{2}$ first decreases with small $d\left(d<d_{0}\right)$ and then increases slowly. It means that, with a bounded synchronized region, the synchronizability is enhanced firstly and then slowly gets weakened after reaching the maximum. The synchronizability of two-layer networks is maximized at $d_{0}=\frac{a \pi^{2} K(K+1)(K+2)}{12 N^{2}}$.

(3) Let $N=200, d=5, a=0.5$, the relationship between the synchronizability and the node degree $K$ is shown in Figures $\mathbf{3 A}, \mathbf{B}$. When the synchronized region is unbounded, as shown in Figure $\mathbf{3 A}, \lambda_{2}$ increases sharply with increasing $K\left(50<K<K_{0}=77\right)$, and then reaches a $\lambda_{2}=$ $2 d=10$. This implies that the synchronizability of twolayer networks is first enhanced and enhanced and then kept invariant. When the synchronized region is bounded, it can be observed from from Figure $3 \mathbf{B}$ that the eigenratio $r=\lambda_{\max } / \lambda_{2}$ first decreases with increasing $K(50<K<$ $\left.K_{0}=77\right)$ and then increases monotonically. It means that the synchronizability is enhanced sharply with increasing $K$, then reaches its maximum, and finally gets weakened. The two-layer networks is maximized at $K_{0}=77$.

(4) Let $K=50, a=d=1$, the relationship between the synchronizability and the network size $N$ is shown in Figures 3C,D. When $300<N<330$, it depicts that $\lambda_{2}$ and the eigenratio $r=\lambda_{\max } / \lambda_{2}$ remain invariant with increasing $N$. When $N>330, \lambda_{2}$ decreases with increasing $N$, and the eigenratio $r=\lambda_{\max } / \lambda_{2}$ increases with increasing $N$. This implies that, with unbounded or bounded synchronized regions, the synchronizability is first kept invariant and then gets weakened with increasing the network size $N$. 

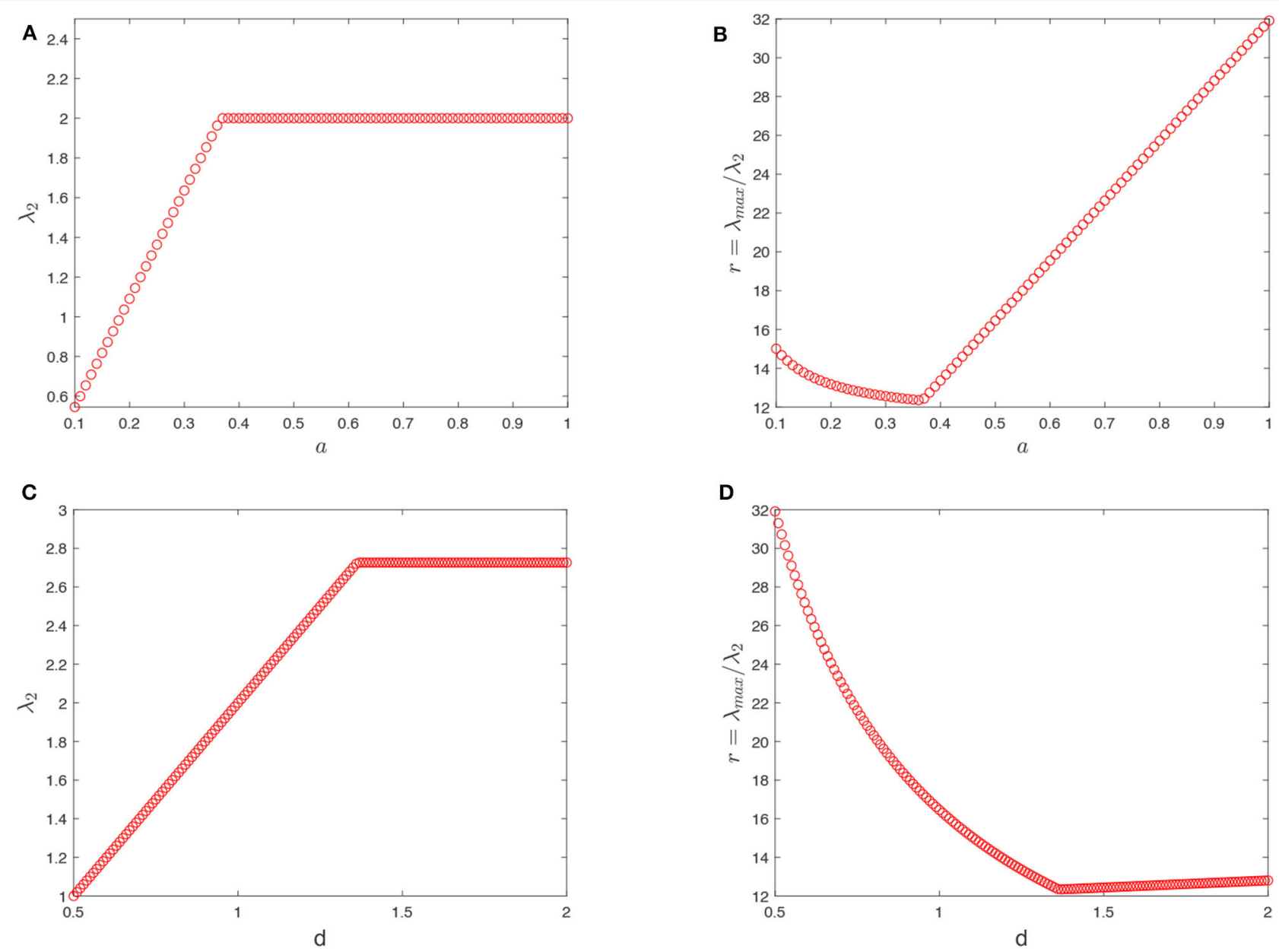

FIGURE 2 | Synchronizability of two-layer K-nearest-neighbor networks. Panels (A,B) display $\lambda_{2}$ and $r=\lambda_{\max } / \lambda_{2}$ vs. the intra-layer coupling strength a ( $N=200$, $K=50, d=1)$. Panels (C,D) display $\lambda_{2}$ and $r=\lambda_{\max } / \lambda_{2}$ vs. the inter-layer coupling strength $d(N=200, K=50, a=0.5)$.

\subsection{The Synchronizability of Multilayer Networks}

Here, we investigate the synchronizability of multilayer K-nearest-neighbor networks. As shown in Supplementary Figures 2,3, the impact of the intra-layer coupling strength $a$, the inter-layer coupling strength $d$, the node degree $K$ and the network size $N$ on network synchronizability are similar to the two-layer K-nearest-neighbor networks.

It can be seen from Supplementary Figure 2 that $\lambda_{2}$ increases nearly linearly at the beginning and then reaches a upper bounded value, the eigenratio $r=\lambda_{\max } / \lambda_{2}$ first decreases and then increases monotonically. It reveals that the synchronizability is enhanced firstly, and then reaches its maximum. Furthermore, the optimal parameters $a_{0}=\frac{6 M N^{2} d}{\pi^{2} K(K+1)(K+2)} \approx 1.8339$, $d_{0}=\frac{a \pi^{2} K(K+1)(K+2)}{6 M N^{2}} \approx 0.2726$ and $K_{0}=133$ are obtained to maximize the synchronizability of multilayer networks. Supplementary Figures 3A,B depict that $\lambda_{2}$ and the eigenratio $r=\lambda_{\max } / \lambda_{2}$ remain invariant with increasing $N(100<$ $N<147)$. When $N>147, \lambda_{2}$ decreases and the eigenratio $r=\lambda_{\max } / \lambda_{2}$ increases with increasing $N$. This implies that, the synchronizability is first kept invariant and then gets weakened with increasing the network size $N$. Let $N=200, K=$ 50, $a=d=0.5$, Supplementary Figures 3C,D show that $\lambda_{2}$ increases at the beginning and then keeps invariant, the eigenratio $r=\lambda_{\max } / \lambda_{2}$ first decreases and then increases slowly with increasing the number of layers $M$. The observation reveals that the synchronizability of multilayer networks is maximized at $M_{0}=6$.

\section{CONCLUSION}

This paper aims to investigate the synchronizability of multilayer networks with K-nearest-neighbor topologies. The master stability function method allows one to analyze how various topological parameters influence network synchronizability. Here, the analytical expressions for the eigenvalues of twolayer and multilayer K-nearest-neighbor networks have been obtained. Further, we have discussed the impacts of the network size, the node degree, the number of layers, the intra-layer 

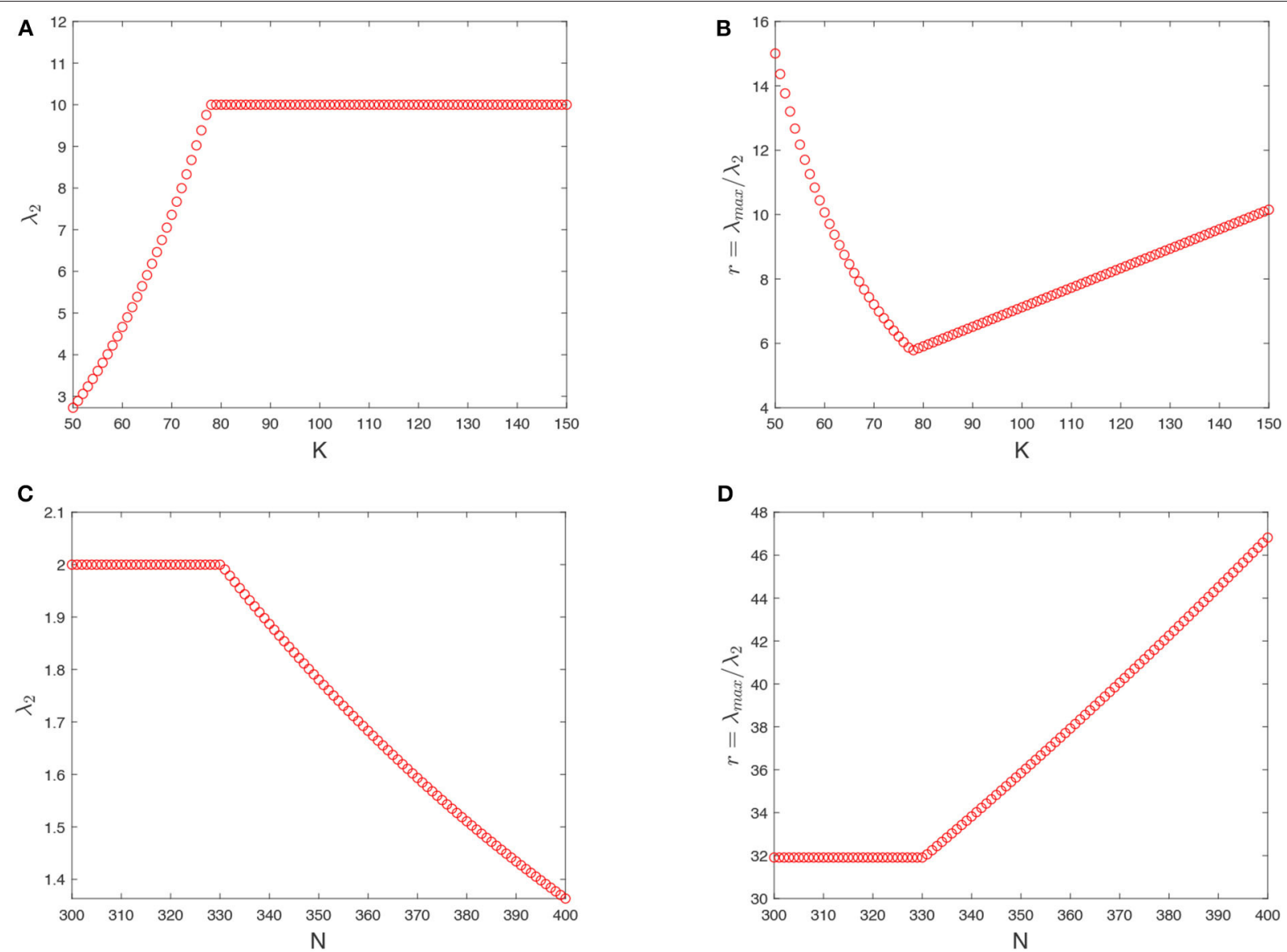

FIGURE 3 | Synchronizability of two-layer K-nearest-neighbor networks. Panels (A,B) display $\lambda_{2}$ and $r=\lambda_{\max } / \lambda_{2}$ vs. the node degree $K(N=200, d=5, a=0.5)$. Panels (C,D) display $\lambda_{2}$ and $r=\lambda_{\max } / \lambda_{2}$ vs. the network size $N(K=50, a=d=1)$.

and the inter-layer coupling strengths on the synchronizability of multilayer K-nearest-neighbor networks. Recently, network coherence $[33,34]$ is an interesting but challenging topic, and coherence analysis of multilayer networks is a part of our future work.

\section{DATA AVAILABILITY STATEMENT}

All datasets generated for this study are included in the article/Supplementary Material.

\section{AUTHOR CONTRIBUTIONS}

LZ and YW contributed to the conception and design of the study. YW organized the literature. LZ performed the design of figures and wrote the first draft of the manuscript. All authors contributed to the manuscript revision and read and approved the submitted version.

\section{FUNDING}

This work was supported by the National Natural Science Foundation of China (Grant No. 61304173), and Foundation of Liaoning Educational Committee (Grant No. LJ2017QL021).

\section{SUPPLEMENTARY MATERIAL}

The Supplementary Material for this article can be found online at: https://www.frontiersin.org/articles/10.3389/fphy. 2020.571507/full\#supplementary-material

\section{REFERENCES}

1. Watts DJ, Strogatz SH. Collective dynamics of 'smallworld' networks. Nature. (1998) 393:440-2. doi: 10.1038/

2. Barabási AL, Albert R. Emergence of scaling in random networks. Science. (1999) 286:509-12. doi: 10.1126/science.286.5439.509

3. Boccaletti S, Latora V, Moreno Y, Chavez M, Hwang DU. Complex networks: structure and dynamics. Phys Rep. (2006) 424:175-308. doi: 10.1016/j.physrep.2005.10.009 
4. Newman MEJ. Networks: An Introduction. Oxford: Oxford University Press (2010).

5. Mucha PJ, Richardson T, Macon K, Porter MA, Onnela JP. Community structure in time-dependent, multiscale, and multiplex networks. Science. (2010) 328:876-8. doi: 10.1126/science.1184819

6. Lee K, Kim JY, Lee S, Goh K. Multiplex networks. In: D’Agostino G, Scala A, editors. Networks of Networks: The Last Frontier of Complexity. Berlin: Springer International Publishing (2014). p.53-72.

7. Kivelä M, Arenas A, Barthelemy M, Gleeson JP, Moreno Y, Porter MA. Multilayer networks. J Complex Netw. (2014) 2:203-71. doi: 10.1093/comnet/cnu016

8. Boccaletti S, Bianconi G, Criado R, del Genio CI, Gómez-Gardeñes J, Romance $\mathrm{M}$, et al. The structure and dynamics of multilayer networks. Physics Reports. (2014) 544:1-C122. doi: 10.1016/j.physrep.2014.07.001

9. Wang XF, Chen GR. Synchronization in small-world dynamical networks. Int J Bifurcat Chaos. (2002) 12:187-92. doi: 10.1142/S0218127402004292

10. Wang XF, Chen GR. Synchronization in scale-free dynamical networks: robustness and fragility. IEEE Trans Circ Syst I Fundament Theory Appl. (2002) 49:54-62. doi: 10.1109/81.974874

11. Arenas A, Díaz-Guilera A, Kurths J, Moreno Y, Zhou CS. Synchronization in complex networks. Phys Rep. (2008) 469:93-153. doi: 10.1016/j.physrep.2008.09.002

12. Li CP, Sun WG, Kurths J. Synchronization between two coupled complex networks. Physical Review E. (2007) 76:046204. doi: 10.1103/PhysRevE.76.046204

13. Yu WW, Chen GR, Lü JH, Kurths J. Synchronization via pinning control on general complex networks. SIAM J Control Optimizat. (2013) 51:1395-416. doi: $10.1137 / 100781699$

14. Xu J, Li N, Zhang XL, Qin XL. Fuzzy synchronization control for fractionalorder chaotic systems with different structures. Front Phys. (2020) 8:155. doi: 10.3389/fphy.2020.00155

15. Liu XY, Lam J, Yu WW, Chen GR. Finite-time consensus of multiagent systems with a switching protocol. IEEE Trans Neural Netw Learn Syst. (2016) 27:853-62. doi: 10.1109/TNNLS.2015.2425933

16. Liu XY, Su HS, Chen MZQ. A switching approach to designing finitetime synchronization controllers of coupled neural networks. IEEE Trans Neural Netw Learn Syst. (2016) 27:471-82. doi: 10.1109/TNNLS.2015.2 448549

17. Gómez S, Diaz-Guilera A, Gómez-Gardeñes J, Perez-Vicente CJ. Diffusion dynamics on multiplex networks. Phys Rev Lett. (2013) 110:028701. doi: 10.1103/PhysRevLett.110.028701

18. Solé-Ribalta A, De Domenico M, Kouvaris NE, Diaz-Guilera A, Gomez S, Arenas A. Spectral properties of the Laplacian of multiplex networks. Phys Rev E. (2013) 88:032807. doi: 10.1103/PhysRevE.88.032807

19. Aguirre J, Sevilla-Escoboza R, Gutiérrez R, Papo D, Buldú JM. Synchronization of interconnected networks: the role of connector nodes. Phys Rev Lett. (2014) 112:248701. doi: 10.1103/PhysRevLett.112.2 48701

20. Gambuzza LV, Frasca M, Gómez-Gardeñes J. Intra-layer synchronization in multiplex networks. Europhys Lett. (2015) 110:20010. doi: $10.1209 / 0295-5075 / 110 / 20010$
21. Sevilla-Escoboza R, Sendiña-Nadal I, Leyva I, Gutierrez R, Buldu JM, Boccaletti S. Inter-layer synchronization in multiplex networks of identical layers. Chaos. (2016) 26:065304. doi: 10.1063/1.4952967

22. Leyva I, Sevilla-Escoboza R, Sendiña-Nadal I, Gutierrez R, Buldu JM, Boccaletti S. Inter-layer synchronization in non-identical multi-layer networks. Sci Rep. (2017) 7:45475. doi: 10.1038/srep45475

23. Wei X, Wu XQ, Lu JA, Zhao JC. Counterpart synchronization of duplex networks with delayed nodes and noise perturbation. J Stat Mech Theory Exp. (2015) 2015:P11021. doi: 10.1088/1742-5468/2015/11/P11021

24. Ning D, Wu XQ, Lu JA, Lü JH. Driving-based generalized synchronization in two-layer networks via pinning control. Chaos. (2015) 25:113104. doi: 10.1063/1.4935069

25. Tang LK, Wu XQ, Lü JH, Lu JA, D’Souza RM. Master stability functions for complete, intralayer, and interlayer synchronization in multiplex networks of coupled Rössler oscillators. Phys Rev E. (2019) 99:012304. doi: 10.1103/PhysRevE.99.012304

26. Wei J, Wu XQ, Lu JA, Wei X. Synchronizability of duplex regular networks. Europhys Lett. (2017) 120:20005. doi: 10.1209/0295-5075/120/20005

27. Xu MM, Lu JA, Zhou J. Synchronizability and eigenvalues of two-layer star networks. Acta Phys Sin. (2016) 65:028902. doi: 10.7498/aps.65.028902

28. Sun J, Li XX, Zhang JH, Shen YZ, Li YY. Synchronizability and eigenvalues of multilayer star networks through unidirectionally coupling. Acta Phys Sin. (2017) 66:188901 (in Chinese). doi: 10.7498/aps.66.188901

29. Deng Y, Jia Z, Deng GM, Zhang QF. Eigenvalue spectrum and synchronizability of multiplex chain networks. Phys A. (2020) 537:122631. doi: 10.1016/j.physa.2019.122631

30. Deng Y, Jia Z, Yang FM. Synchronizability of multilayer star and star-ring networks. Discrete Dyn Nat Soc. (2020) 2020:9143917. doi: $10.1155 / 2020 / 9143917$

31. Pecora LM, Carroll TL. Master stability functions for synchronized coupled systems. Phys Rev Lett. (1998) 80:2109. doi: 10.1103/PhysRevLett.80.2109

32. Wang XF, Li X, Chen GR. Network Science: An Introduction. Beijing: High Education Press (2012) (in Chinese).

33. Hong MD, Sun WG, Liu SY, Xuan TF. Coherence analysis and Laplacian energy of recursive trees with controlled initial states. Front Inf Technol Electron Eng. (2020) 21:931-8. doi: 10.1631/FITEE.1900133

34. Sun WG, Sun MT, Guan JB, Jia Q. Robustness of coherence in noisy scale-free networks and applications to identification of influential spreaders. IEEE Trans Circ Syst II Express Briefs. (2020) 67:1274-8. doi: 10.1109/TCSII.2019.2929139

Conflict of Interest: The authors declare that the research was conducted in the absence of any commercial or financial relationships that could be construed as a potential conflict of interest.

Copyright (C) 2020 Zhang and Wu. This is an open-access article distributed under the terms of the Creative Commons Attribution License (CC BY). The use, distribution or reproduction in other forums is permitted, provided the original author(s) and the copyright owner(s) are credited and that the original publication in this journal is cited, in accordance with accepted academic practice. No use, distribution or reproduction is permitted which does not comply with these terms. 